\title{
Optimal cloning with respect to the relative error
}

\author{
Alexey E. Rastegin \\ Department of Theoretical Physics, Irkutsk State University, Gagarin Bv. 20, Irkutsk 664003, Russia
}

\begin{abstract}
The relative error of cloning of quantum states with arbitrary prior probabilities is considered. It is assumed that the ancilla may contain some a priori information about the input state to be cloned. The lower bound on the relative error for general cloning scenario is derived. Both the case of two-state set and case of multi-state set are analyzed in details. The treated figure of merit is compared with other optimality criteria. The quantum circuit for optimal cloning of a pair of pure states is constructed.
\end{abstract}

PACS numbers: 03.65.ta, 03.67-a

Keywords: State-dependent quantum cloning; stronger no-cloning theorem; distinguishability transfer gate.

\section{INTRODUCTION}

The quantum information topics are the subject of active research [1]. The impressive progress have been reached in the quantum cryptography [2] and study of quantum algorithms [3]. Due to an impact on security in quantum cryptography, the quantum cloning is still a significant topic. At the same time, a cloning itself is hardly sufficient for an eavesdropping [4, [5]. No-copying results have been established for pure states [6, 7] as well as for mixed states [8]. In view of such evidences, the question arose how well quantum cloning machines could work. In effect, the basic importance of the no-cloning theorem is expressed much better in more detailed results, which also give explicit bounds on an amount of the noise.

After the seminal work by Bužek and Hillery [9], many approaches to approximate quantum cloning have been developed. In view of existing reviews [10, 11], we cite only the literature that is directly connected to our results. An approximate cloning of two prescribed pure states was first considered in Ref. [12]. This kind of cloning operation is usually referred to as state-dependent cloning [13]. In general, various types of state-dependent cloners may be needed with respect to the question of interest [14, 15]. Errors inevitably occur already in a cloning of two nonorthogonal states [12]. How close to perfection can a cloning be? Of course, any explicit answer must utilize some optimality criterion. We will refer criterion used in Ref. [12] to as the absolute error [16]. Chefles and Barnett [17] derived the least upper bound on the global fidelity for cloning of two pure states with arbitrary prior probabilities. The quantum circuit that reaches this upper bound was also constructed [17]. The global fidelity of cloning of several equiprobable pure states was examined in Ref. [18].

Although cloning problems were mostly analyzed with respect to the fidelity criteria, other measures of closeness of quantum states are relevant. For example, the "partial" quantum cloning is easier to analyze with respect to the squared Hilbert-Schmidt distance [19]. One of criteria, relative error [16], has been shown to be useful within the B92 protocol emerged in Ref. [20]. Deriving bounds on the relative error was based on the spherical triangle inequality [16] and the notion of the angle [21] sometimes called the Bures length 22]. Using this new method, a cloning of two equiprobable mixed states was studied with respect to the global fidelity [21]. The results of Ref. [16] were partially extended to mixed-state cloning [23].

In a traditional approach, the ancilla does not contain a priori information of state to be cloned just now. A more general case is the scope of the stronger no-cloning theorem [24]. Namely, a perfect cloning is achievable, if and only if the full information of the clone has already been provided in the ancilla state alone. In Ref. [25] we examined a cloning of finite set of states when the ancilla contains a partial information of the input state. So, the previous result of Ref. [17] was extended to both the mixed states and a priori information.

In this paper, we study the relative error of cloning of several mixed states, having arbitrary prior probabilities. $A$ priori information in the ancilla is also assumed. In Section II, the relative error criterion introduced in Refs. [16, 23] is extended to the general cloning scenario. We derive the lower bounds on the relative error for cloning of two-state set (see Section III) and multi-state set (see Section IV). In Section V, the relative error is compared with other optimality criteria. We also build the quantum circuit for cloning of two pure states (see Section VI). This circuit reaches the lower bound on the relative error for arbitrary prior probabilities and a priori knowledge about the input. Section VII concludes the paper. 


\section{RELATIVE ERROR OF CLONING}

The main problem posed formally is this. We have $N$ indistinguishable $n$-level systems that which are all prepared in the same state $\rho_{j} \in\left\{\rho_{1}, \ldots, \rho_{m}\right\}$ from the known set of density operators on the space $\mathcal{V}=\mathbb{C}^{n}$. These $N$ systems form the register $B$. Its initial state is a density operator $\varrho_{j}=\rho_{j}^{\otimes N}$ on the input Hilbert space $\mathcal{H} \equiv \mathcal{V} \otimes N$. The prior probabilities $p_{j}$ of states $\varrho_{j}$ obey the normalization condition $\sum_{j=1}^{m} p_{j}=1$. We aim to get a larger number $L$ of copies of the given $N$ originals by means of the ancilla whose initial state is $\Upsilon_{j}$ according to the input $\varrho_{j}$. Here we mean a system $C E$ composed of extra register $C$ and environment $E$. The extra register $C$ contains $M=L-N$ additional $n$-level systems, each is to receive the clone of $\rho_{j}$. If we include an environment space then any deterministic physical operation may be expressed as a unitary evolution. Thus, the final state of two registers $B C$ is the partial trace over environment space

$$
\varrho_{j}^{\prime}=\mathcal{E}\left(\varrho_{j}\right)=\operatorname{Tr}_{E}\left[\mathrm{U}\left(\varrho_{j} \otimes \Upsilon_{j}\right) \mathrm{U}^{\dagger}\right]
$$

The output $\varrho_{j}^{\prime}$ is a density operator on the output Hilbert space $\mathcal{H}^{\prime} \equiv \mathcal{V}^{\otimes L}$.

The actual output $\varrho_{j}^{\prime}$ must be compared with the ideal output $\rho_{j}^{\otimes L}$. Many measures of distinguishability between mixed quantum states are based on the fidelity [26]. We shall employ the angles and the sine metric [27]. Let $|A|$ denote a unique positive square root of $\mathrm{A}^{\dagger} \mathrm{A}$. The fidelity between the two density operators $\sigma$ and $\omega$ is equal to $F(\omega, \sigma)=(\operatorname{Tr}|\sqrt{\omega} \sqrt{\sigma}|)^{2}[28,29]$. In terms of this measure, the angle $\Delta(\omega, \sigma) \in[0 ; \pi / 2]$ between $\sigma$ and $\omega$ is defined by the equality $\cos ^{2} \Delta(\omega, \sigma)=F(\omega, \sigma)[21]$. It is also referred to as the Bures length [22], because of its close relation to the standard Bures metric $d_{B}(\omega, \sigma)=(2-2 \sqrt{F}(\omega, \sigma))^{1 / 2}$. Due to the spherical triangle inequality [21],

$$
\Delta(\omega, \sigma) \leq \Delta(\omega, \eta)+\Delta(\sigma, \eta)
$$

We introduce the sine distance [27] between $\omega$ and $\sigma$ as $d(\omega, \sigma):=\sin \Delta(\omega, \sigma)$. This metric on the space of quantum states has a close relation to the trace distance [30] and enjoys the following [23, 27]. For any POVM measurement $\left\{\mathrm{A}_{\mu}\right\}$, there holds

$$
\left|\operatorname{Tr}\left(\mathrm{A}_{\mu} \omega\right)-\operatorname{Tr}\left(\mathrm{A}_{\mu} \sigma\right)\right| \leq d(\omega, \sigma)
$$

Here $\operatorname{Tr}\left(\mathrm{A}_{\mu} \omega\right)$ is the probability of obtaining outcome $\mu$, if the state right before measurement was $\omega$. A more detailed characterization of such a kind can be posed via majorization relations [31]. We also have $|F(\omega, \eta)-F(\sigma, \eta)| \leq d(\omega, \sigma)$. Since the fidelity function cannot decrease under any deterministic quantum operation [8], the last inequality can be extended to

$$
\left|F\left(\mathcal{E}(\omega), \eta^{\prime}\right)-F\left(\mathcal{E}(\sigma), \eta^{\prime}\right)\right| \leq d(\omega, \sigma)
$$

Using the sine distance is reasonable approach due to the inequalities (2.3) and (2.4). For brevity, let us denote

$$
\delta_{j}^{\prime} \equiv \Delta\left(\varrho_{j}^{\prime}, \rho_{j}^{\otimes L}\right), \quad \Delta_{j k}^{(L)} \equiv \Delta\left(\rho_{j}^{\otimes L}, \rho_{k}^{\otimes L}\right) .
$$

When two inputs $\varrho_{+}$and $\varrho_{-}$are equiprobable, the relative error is defined by $R_{N L}=\left(\sin \delta_{+}^{\prime}+\sin \delta_{-}^{\prime}\right) / \sin \Delta_{ \pm}^{(L)}$ [16, 23]. Meaning $p_{ \pm}=1 / 2$, it can be rewritten as

$$
R_{N L}=\sum_{j= \pm} p_{j} \frac{\sin \delta_{j}^{\prime}}{(1 / 2) \sin \Delta_{ \pm}^{(L)}} \equiv \sum_{j= \pm} p_{j} \frac{d\left(\varrho_{j}^{\prime}, \rho_{j}^{\otimes L}\right)}{(1 / 2) d\left(\rho_{+}^{\otimes L}, \rho_{-}^{\otimes L}\right)}
$$

The right-hand side of Eq. (2.6) is quite relevant to the case of arbitrary prior probabilities. Since the distance $d\left(\varrho_{j}^{\prime}, \rho_{j}^{\otimes L}\right)$ estimates the difference between two probability distributions (see Eq. (2.3) $)$, a reliable identification of original input via measurement over clones may be provided only when

$$
d\left(\varrho_{+}^{\prime}, \rho_{+}^{\otimes L}\right)+d\left(\varrho_{-}^{\prime}, \rho_{-}^{\otimes L}\right) \ll d\left(\rho_{+}^{\otimes L}, \rho_{-}^{\otimes L}\right) .
$$

So we see a reason for using a ratio of $d\left(\varrho_{j}^{\prime}, \rho_{j}^{\otimes L}\right)$ just to the half of $d\left(\rho_{+}^{\otimes L}, \rho_{-}^{\otimes L}\right)$. In addition, this choice implies that the tight lower bound on relative error generally recovers the range $[0 ; 1]$.

We shall now extend the notion of relative error for the set $\mathfrak{S}=\left\{\rho_{1}, \ldots, \rho_{m}\right\}$ with $m>2$, when the number of different pairs is equal to $m(m-1) / 2$. The probability of taking the pair $\pi_{j k}=\left\{\rho_{j}, \rho_{k}\right\}$ is equal to

$$
q_{j k}=p_{j} p_{k}\left(\sum_{1 \leq j<k \leq m} p_{j} p_{k}\right)^{-1}
$$


where $\sum_{1 \leq j<k \leq m} q_{j k}=1$. We clearly have $p_{j}=1 / m$ and $q_{j k}=2 /(m(m-1))$ for the set of $m$ equiprobable states. To each pair $\pi_{j k}$ assign the quantity

$$
R_{N L}\left(\pi_{j k}\right)=2\left\{p_{j} d\left(\varrho_{j}^{\prime}, \rho_{j}^{\otimes L}\right)+p_{k} d\left(\varrho_{k}^{\prime}, \rho_{k}^{\otimes L}\right)\right\} /\left\{\left(p_{j}+p_{k}\right) d\left(\rho_{j}^{\otimes L}, \rho_{k}^{\otimes L}\right)\right\},
$$

which takes into account that, perhaps, $p_{j}+p_{k} \neq 1$. It is natural to put the weighted average of the $m(m-1) / 2$ quantities (2.8).

Definition 1. The relative error of $N \rightarrow L$ cloning of the set $\mathfrak{S}=\left\{\rho_{1}, \ldots, \rho_{m}\right\}$ is defined by

$$
R_{N L}(\mathfrak{S}):=\sum_{1 \leq j<k \leq m} q_{j k} R_{N L}\left(\pi_{j k}\right) .
$$

Let the prior probability be value of order $\epsilon \ll 1$ for all the states except $\rho_{1}$ and $\rho_{2}$. That is, we take $p_{j}=O(\epsilon)$ for $j \neq 1,2$, whence $q_{12}=1+O(\epsilon), q_{j k}=O(\epsilon)$ for the rest pairs. The expression (2.9) for relative error is simply reduced to $R_{N L}(\mathfrak{S})=R_{N L}\left(\pi_{12}\right)+O(\epsilon)$. In the same manner, we can find $R_{N L}(\mathfrak{S})=R_{N L}(\mathfrak{T})+O(\epsilon)$, when $\mathfrak{T} \subset \mathfrak{S}$ and probabilities $p_{j}=O(\epsilon)$ except for the states $\rho_{j} \in \mathfrak{T}$ solely.

We are interested in a nontrivial lower bound on the relative error (2.9). Our approach to obtaining the limits utilizes triangle inequalities [16, 21]. Following the method, we shall derive the angle relation from which bound on the relative error is simply obtained. It is handy to introduce the angle $\varkappa_{j k} \in[0 ; \pi / 2]$ as

$$
\varkappa_{j k}:=\arccos \sqrt{F\left(\rho_{j}^{\otimes N}, \rho_{k}^{\otimes N}\right) F\left(\Upsilon_{j}, \Upsilon_{k}\right)} .
$$

The laws of quantum theory impose some restrictions on acceptable values of angles $\delta_{j}^{\prime}$, whence nontrivial bounds for different figures of cloning merit follow.

\section{LOWER BOUND FOR TWO-STATE SET}

In the case of the two-state set $\left\{\rho_{+}, \rho_{-}\right\}$, the initial state of ancilla is $\Upsilon_{+}$or $\Upsilon_{-}$according to the input which is $\rho_{+}$ or $\rho_{-}$. We further assume that

$$
F\left(\Upsilon_{+}, \Upsilon_{-}\right)>F\left(\rho_{+}^{\otimes M}, \rho_{-}^{\otimes M}\right)
$$

and, by the multiplicativity of fidelity, $\cos \varkappa_{ \pm}>F\left(\rho_{+}^{\otimes L}, \rho_{-}^{\otimes L}\right)$. The motivation is as follows. If the inequality (3.1) is not satisfied then there are states sufficient for perfect cloning [23, 25]. That is, there exist states $\Upsilon_{+}$and $\Upsilon_{-}$such that $\rho_{j}^{\otimes M}=\operatorname{Tr}_{E}\left(\Upsilon_{j}\right)$. Hence we can mention a trivial bound $R_{N L} \geq 0$ only. So we presuppose that the inequality (3.1) is valid. As result, we have

$$
\Delta_{ \pm}^{(N)} \leq \varkappa_{ \pm}<\Delta_{ \pm}^{(L)}
$$

With no loos of generality, we assume that $p_{+} \geq p_{-}$.

Theorem 2 The relative error $R_{N L}$ of cloning of the set $\left\{\rho_{+}, \rho_{-}\right\}$satisfies

$$
R_{N L} \geq 2 p_{-} \sin \left(\Delta_{ \pm}^{(L)}-\varkappa_{ \pm}\right) \sin ^{-1} \Delta_{ \pm}^{(L)} .
$$

Proof Applying the inequality (2.2) twice, we obtain

$$
\Delta_{ \pm}^{(L)} \leq \Delta\left(\rho_{+}^{\otimes L}, \varrho_{+}^{\prime}\right)+\Delta\left(\rho_{-}^{\otimes L}, \varrho_{+}^{\prime}\right) \leq \delta_{+}^{\prime}+\delta_{-}^{\prime}+\Delta\left(\varrho_{+}^{\prime}, \varrho_{-}^{\prime}\right) .
$$

Recall that the fidelity function is multiplicative, preserved by unitary evolution and non-decreasing under the operation of partial trace [8, 29]. So we obtain

$$
F\left(\rho_{+}^{\otimes N}, \rho_{-}^{\otimes N}\right) F\left(\Upsilon_{+}, \Upsilon_{-}\right) \leq F\left(\varrho_{+}^{\prime}, \varrho_{-}^{\prime}\right),
$$

whence $\Delta\left(\varrho_{+}^{\prime}, \varrho_{-}^{\prime}\right) \leq \varkappa_{ \pm}$. Combining this with Eq. (3.4) provides

$$
\delta_{+}^{\prime}+\delta_{-}^{\prime} \geq \Delta_{ \pm}^{(L)}-\varkappa_{ \pm} .
$$

Consider the function $g\left(\delta_{+}^{\prime}, \delta_{-}^{\prime}\right):=p_{+} \sin \delta_{+}^{\prime}+p_{-} \sin \delta_{-}^{\prime}$ to be minimized. We want to minimize $g\left(\delta_{+}^{\prime}, \delta_{-}^{\prime}\right)$ under the constraint (3.5), $0 \leq \delta_{+}^{\prime} \leq \pi / 2$ and $0 \leq \delta_{-}^{\prime} \leq \pi / 2$. This task is solved in Appendix A. By substitutions, we then have

$$
\min g\left(\delta_{+}^{\prime}, \delta_{-}^{\prime}\right)=p_{-} \sin \left(\Delta_{ \pm}^{(L)}-\varkappa_{ \pm}\right)
$$


and further the statement of Theorem 2.

For equiprobable states, the bound (3.3) is reduced to the lower bound deduced in Ref. [23]. In terms of $f=$ $\sqrt{F}\left(\rho_{+}, \rho_{-}\right)$and $\phi=\sqrt{F}\left(\Upsilon_{+}, \Upsilon_{-}\right)$, we rewrite (3.1) as $\phi>f^{M}$. By $\cos \Delta_{ \pm}^{(L)}=f^{L}$ and cos $\varkappa_{ \pm}=f^{M} \phi$, the bound (3.3) becomes

$$
R_{N L} \geq 2 p_{-}\left\{f^{M} \phi-f^{L} \sqrt{\left(1-f^{2 M} \phi^{2}\right) /\left(1-f^{2 L}\right)}\right\}
$$

At fixed $f$ and $\phi$, the right-hand side of Eq. (3.7) is an increasing function of probability $p_{-}$. That is, it decreases as the prior probabilities differ. This is analog of that the upper bound on the global fidelity increases in such a situation [25]. We are rather interested in dependence of the bound on $\phi$. This parameter marks a top amount of an a priori information, which can initially be laid in the ancilla. The more a value of $\phi$, the less this amount. The angle $\varkappa_{ \pm}$is a decreasing function of $\phi$. In the range (3.2), the lower bound by Theorem 3 is a decreasing function of $\varkappa_{ \pm}$. So the right-hand side of (3.7) increases as the marker $\phi$ of additional information increases. For $\phi=f^{M}$ the perfect cloning can be reached [23, 25]. In line with this fact, we have $\varkappa_{ \pm}=\Delta_{ \pm}^{(L)}$ and the vanishing bound on $R_{N L}$. On the contrary, in the usual cloning there is no a priori information, i.e. $\Upsilon_{+}=\Upsilon_{-}$and $\phi=1$. Then the bound by Theorem 2 reaches its maximum as a function of $\phi$. The above points reproduce the observations of Ref. [23] in more general setting.

If $N \rightarrow \infty$ at fixed $M$ then the right-hand side of the inequality (3.7) goes to zero. This is natural because infinite number $N$ of originals can provide almost perfect cloning. If $M \rightarrow \infty$ at fixed $N$ then the right-hand side of (3.7) recovers the value $2 p_{-} f^{N} \phi$. In the standard cloning of equiprobable states $\left(\phi=1, p_{-}=1 / 2\right)$, this value can be arbitrarily close to 1, since $\sup \left\{2 p_{-} f^{N} \phi: 0 \leq f^{M}<\phi \leq 1\right\}=1$. It is not insignificant that the value $2 p_{-} f^{N} \phi$ gives the minimal size of probability of inconclusive answer for unambiguous discrimination at $p_{-}=1 / 2$. Namely, the success discrimination of the equiprobable pure states $\left|\Omega_{ \pm}\right\rangle=\left|\psi_{ \pm}\right\rangle^{\otimes N} \otimes\left|\theta_{ \pm}\right\rangle$occurs with the optimal probability $\left(1-\left|\left\langle\Omega_{+} \mid \Omega_{-}\right\rangle\right|\right)[32,33]$. The value $2 p_{-} f^{N} \phi$ is obtained for $\left|\left\langle\psi_{+} \mid \psi_{-}\right\rangle\right|=f$ and $\left|\left\langle\theta_{+} \mid \theta_{-}\right\rangle\right|=\phi$. Note that the upper bound on the global fidelity in the limit $M \rightarrow \infty$ at fixed $N$ goes to well-known Helstrom bound [17, 25]. It is the probability of correctly distinguishing between two pure states $\left|\Omega_{ \pm}\right\rangle$by the optimal strategy [34].

\section{LOWER BOUND FOR MULTI-STATE SET}

We now obtain a lower bound on the relative error of cloning of the set $\mathfrak{S}=\left\{\rho_{1}, \ldots, \rho_{m}\right\}$. As before, the prior probabilities are arbitrary and constrained only by the normalization condition. Like (3.2), we have the acceptable range

$$
\Delta_{j k}^{(N)} \leq \varkappa_{j k}<\Delta_{j k}^{(L)}
$$

According to Theorem 2, each term of sum in the right-hand side of (2.9) obeys

$$
R_{N L}\left(\pi_{j k}\right) \geq 2 \min \left\{p_{j}, p_{k}\right\} \sin \left(\Delta_{j k}^{(L)}-\varkappa_{j k}\right)\left(\left(p_{j}+p_{k}\right) \sin \Delta_{j k}^{(L)}\right)^{-1}
$$

Hence the desired bound is established as follows.

Theorem 3 The relative error of $N \rightarrow L$ cloning of the set $\mathfrak{S}=\left\{\rho_{1}, \ldots, \rho_{m}\right\}$ satisfies

$$
R_{N L}(\mathfrak{S}) \geq \sum_{1 \leq j<k \leq m} 2 q_{j k} \frac{\min \left\{p_{j}, p_{k}\right\}}{p_{j}+p_{k}} \frac{\sin \left(\Delta_{j k}^{(L)}-\varkappa_{j k}\right)}{\sin \Delta_{j k}^{(L)}} .
$$

As a straightforward extension, the bound (4.3) succeeds many features of the bound (3.3). If two probabilities, say, $p_{1}$ and $p_{2}$ are variable and the rest of parameters is fixed, then the bound (4.3) decreases as these probabilities differ. If some one probability is close to 1 and other probabilities are small, then the bound is close to zero. This behavior is expected, because single known state can be cloned perfectly. For equal a priori probabilities $p_{j}=1 / m$, the bound by Theorem 3 becomes

$$
R_{N L} \geq \frac{2}{m(m-1)} \sum_{1 \leq j<k \leq m}\left(\cos \varkappa_{j k}-\sin \varkappa_{j k} \cot \Delta_{j k}^{(L)}\right) .
$$

It is natural that both the bounds given by (4.3) and (4.4) decrease as $\varkappa_{j k}$ increases. Indeed, the parameter $\varkappa_{j k}$ characterizes an amount of prior information. If the upper limit of Eq. (4.1) is saturated for some pair $\pi_{j k}$ then corresponding summands in the right-hand sides of Eqs. (4.3) and (4.4) vanish. This is the case of potentially perfect 
cloning. On the whole, these conclusions on a role of a priori information in the ancilla add to the stronger no-cloning theorem.

A question is, whether the lower bounds (3.3) and (4.3) can be reached? In general, it is not the case, though the bound by Theorem 3 is least for two pure states. The quantum circuit for optimal cloning will be built in the next section. The subject matter changes for $m>2$. From the viewpoint of minimization the bound of Theorem 3 is approximate. As reasons of Appendix A show, saturating the inequality (4.2) holds if and only if $\delta_{j}^{\prime}=0$, $\delta_{k}^{\prime}=\Delta_{j k}^{(L)}-\varkappa_{j k}$ for $p_{j} \geq p_{k}$ (for $p_{j} \leq p_{k}$ the angles $\delta_{j}^{\prime}$ and $\delta_{k}^{\prime}$ should be swapped in the two equalities). These two equalities per each of $m(m-1) / 2$ pairs totally give $m(m-1)$ conditions. For saturating Eq. (4.3), $m$ variables $\delta_{j}^{\prime}$ must satisfy all these $m(m-1)$ conditions. Except for some special cases, this is not possible. Thus, the presented limit is somewhat rough.

More rigorous way may be as follows. Similar to (3.5), we have arrived at the $m(m-1) / 2$ inequalities of a kind $\delta_{j}^{\prime}+\delta_{k}^{\prime} \geq \Delta_{j k}^{(L)}-\varkappa_{j k}$. Together with the $m$ conditions $0 \leq \delta_{j}^{\prime} \leq \pi / 2$, these relations specify some simplex in $m$-dimensional real space. The relative error (2.9) can be rewritten in the form

$$
R_{N L}(\mathfrak{S})=2 \sum_{j=1}^{m} r_{j} p_{j} \sin \delta_{j}^{\prime}
$$

where $r_{j}=\sum_{k \neq j} q_{j k}\left(\left(p_{j}+p_{k}\right) \sin \Delta_{j k}^{(L)}\right)^{-1}$. The task is to minimize the function (4.5) in the above simplex. So we come across a difficult problem of nonlinear programming (the simple case $m=2$ of this problem is considered in Appendix A). For $0 \leq \delta_{j}^{\prime} \leq \pi / 2$, the minimized function is concave. So the problem of minimization is reduced to finding extremal points of the simplex. If the values of parameters are prescribed, the wanted minimum can be found numerically. At the same time, it is complicated to obtain an explicit formula for general case. But even if we should find it, we still would not have a complete solution to the problem of mixed-state cloning. Indeed, it is not necessary that bound given by such a formula be least. So we have restricted our consideration to obtaining of the bound by Theorem 3. Rough though this bound is, it has straightforward form and allows to estimate how a merit of state-dependent cloning is limited.

\section{COMPARISON OF DIFFERENT CRITERIA}

We shall now expose the relative error in comparison with other optimality criteria. For the sake of simplicity, we restrict to the $N \rightarrow L$ cloning of two equiprobable pure states $\left|\psi_{ \pm}\right\rangle$without a priori information in the ancilla. How able to good cloning is the pair? This question is central to applications of quantum cloning. In principle, we may assume both the deterministic cloning and probabilistic cloning [35]. A merit of deterministic cloning may be viewed with respect to the global fidelity, the absolute error and the relative error. For equiprobable inputs, the global fidelity is expressed by $F_{N L}=2^{-1} \cos ^{2} \delta_{+}^{\prime}+2^{-1} \cos ^{2} \delta_{-}^{\prime}$ [17, 21]. Hillery and Bužek [12] used the measure $A_{N L}=2^{-1} \sin \delta_{+}^{\prime}+2^{-1} \sin \delta_{-}^{\prime}$. This measure will be referred to as absolute error [16]. The relative error is defined by Eq. (2.6). In probabilistic cloning, the exact clone of an input successfully generated with the maximal probability [35, 36]

$$
\max P_{N L}=\left(1-f^{N}\right) /\left(1-f^{L}\right)
$$

where $f$ denote the overlap $\left|\left\langle\psi_{+} \mid \psi_{-}\right\rangle\right|$between states $\left|\psi_{ \pm}\right\rangle$. As it is shown in Refs. [17, 23], the maximum of the global fidelity is equal to

$$
\max F_{N L}=\frac{1}{2}\left(1+f^{L+N}+\sqrt{\left(1-f^{2 N}\right)\left(1-f^{2 L}\right)}\right)
$$

According to (3.7), the minimum of the relative error is reduced to

$$
\min R_{N L}=f^{N}-f^{L}\left(1-f^{2 N}\right)^{1 / 2}\left(1-f^{2 L}\right)^{-1 / 2}
$$

for $\phi=1$ and $p_{ \pm}=1 / 2$. For the absolute error we have 16 ]

$$
\min A_{N L}=f^{N} \sqrt{1-f^{2 L}}-f^{L} \sqrt{1-f^{2 N}} .
$$

Let us consider the two cases: (i) the states are $\left|\psi_{ \pm}\right\rangle$are almost orthogonal, i.e. $f=\varepsilon \ll 1$; (ii) the states are $\left|\psi_{ \pm}\right\rangle$ are almost identical, i.e. $f=1-\epsilon$ with $\epsilon \ll 1$. A behaviour of each of the criteria is shown in Table $1(N<L)$. 
TABLE I: An asymptotic behaviour of the four criteria.

\begin{tabular}{lcr}
\hline \hline Figure of merit & $(\mathrm{i}) f=\varepsilon \ll 1$ & $(\mathrm{ii}) f=1-\epsilon(\epsilon \ll 1)$ \\
\hline $\max F_{N L}=$ & $1-\varepsilon^{2 N} / 4+\cdots$ & $1-(\sqrt{L}-\sqrt{N})^{2} \epsilon / 2+\cdots$ \\
$\min A_{N L}=$ & $\varepsilon^{N}+\cdots$ & $(\sqrt{2 L}-\sqrt{2 N}) \epsilon^{1 / 2}+\cdots$ \\
$\min R_{N L}=$ & $\varepsilon^{N}+\cdots$ & $1-\sqrt{N / L}+(\sqrt{L N}-N) \epsilon+\cdots$ \\
$\max P_{N L}=$ & $1-\varepsilon^{N}+\cdots$ & $N / L-N(L-N) \epsilon /(2 L)+\cdots$ \\
\hline \hline
\end{tabular}

As it is clear from the second column, for the case (i) all the measures endorse a good merit of both the deterministic and probabilistic cloning. In effect, the optimum of global fidelity is close to one, the optimum of absolute and relative error is close to zero. The probability of success is close to one. It is natural because orthogonal states can perfectly be cloned. The principal distinction of the relative error is revealed in the case (ii). It seems offhand that two almost identical states can be cloned very well. Both the global fidelity and absolute error approve the conclusion $\left(\max F_{N L} \approx 1\right.$ and $\left.\min A_{N L} \approx 0\right)$. It would be rash to accept this. In effect, the optimal probability max $P_{N L}$ is generally not close to one. The first term $N / L$ is almost one only if the number $M=L-N$ of actual clone is negligible in comparison with the number $N$ of originals. In line with this, the optimum of relative error is close to zero for $N / L \approx 1$. But the probability is close to zero and the relative error is close to one when the number $M=L-N$ of actual clone is large. We see that both the global fidelity and absolute error lose sight of the important aspect of deterministic cloning. Even for the primary $1 \rightarrow 2$ cloning, we have $\max P_{N L}=1 / 2-\epsilon / 4+\cdots$ and $\min R_{N L}=1-2^{-1 / 2}+O(\epsilon) \approx 0.3+O(\epsilon)$, that is both the probabilistic and deterministic strategies are restricted enough. In contrast with the global fidelity and the absolute error, for the case (ii) a behaviour of relative error is crucially dependent on numbers $N$ and $L$. Similar to the optimal probability of success, the criterion of relative error emphasizes that any cloning is not isolated stage in quantum information processing. As a rule, the outputs of cloning machine are subjects of further operations, say, a discrimination. For example, in the cryptographic B92 scheme Alice encodes the bits into two non-orthogonal pure states [20]. So Bob can apply the unambiguous discrimination [4]. But the closer used states are to each other the larger number of discarded bits is in the total sequence. On the other hand, a sufficiently great closeness of the used states will prevent the eavesdropping. Unlike both the global fidelity and absolute error, the notion of relative error allows to take such aspects into account.

\section{CIRCUIT FOR OPTIMAL CLONING OF PURE STATES}

We shall now build quantum circuits for the optimal relative-error cloning of two pure states $\left|\psi_{ \pm}\right\rangle$with arbitrary prior probabilities $p_{ \pm}\left(p_{+} \geq p_{-}\right)$. A priori information about actually input state is contained in the state of ancilla which is either $\left|\theta_{+}\right\rangle$or $\left|\theta_{-}\right\rangle$. Without loss of generality, we take the product $\left\langle\psi_{+} \mid \psi_{-}\right\rangle$to be positive real. These states are parametrized as $\left|\psi_{ \pm}\right\rangle=\cos \alpha_{0}|0\rangle \pm \sin \alpha_{0}|1\rangle \equiv\left|\varphi_{ \pm}\left(\alpha_{0}\right)\right\rangle,\left|\theta_{ \pm}\right\rangle=\cos \theta|0\rangle \pm \sin \theta|1\rangle \equiv\left|\varphi_{ \pm}(\theta)\right\rangle$. The overlap is $\left\langle\psi_{+} \mid \psi_{-}\right\rangle=\cos 2 \alpha_{0}$ with $\alpha_{0} \in(0 ; \pi / 4)$. So, we have the register of $(L+1)$ qubits, where $M$ qubits are initially in the blank state $|0\rangle, N$ qubits are in the state to be cloned, and one qubit is ancillary. Our aim is to transform these states according to the specification.

The strategy is an extension of the known one [17] and uses the distinguishability transfer gate (see Appendix B). First, the information about the input $N$ originals is transferred into one qubit. We mark the ancillary qubit by " 0 ", the $N$ original qubits by " $1, \ldots, N "$, and the $M$ additional qubits by " $N+1, \ldots, L "$. The just left gate acts on the qubits $(N-1)$ and $N$ as

$$
\mathrm{D}_{N}\left(\alpha_{0}, \alpha_{0}\right)\left|\varphi_{ \pm}\left(\alpha_{0}\right)\right\rangle_{N-1} \otimes\left|\varphi_{ \pm}\left(\alpha_{0}\right)\right\rangle_{N}=\left|\varphi_{ \pm}\left(\alpha_{1}\right)\right\rangle_{N-1} \otimes|0\rangle_{N}
$$

where $\cos 2 \alpha_{1}=\left(\cos 2 \alpha_{0}\right)^{2}$. Then an operation is applied to qubits $(N-2)$ and $(N-1)$, and so on. In the first stage, the gate $\mathrm{D}_{j}$ transfers the distinguishability from $j$ th qubit to $(j-1)$ th $(j$ runs from $N$ to 2$)$, i.e.

$$
\mathrm{D}_{j}\left(\alpha_{0}, \alpha_{N-j}\right)\left|\varphi_{ \pm}\left(\alpha_{0}\right)\right\rangle_{j-1} \otimes\left|\varphi_{ \pm}\left(\alpha_{N-j}\right)\right\rangle_{j}=\left|\varphi_{ \pm}\left(\alpha_{N-j+1}\right)\right\rangle_{j-1} \otimes|0\rangle_{j}
$$

where $\cos 2 \alpha_{0} \cos 2 \alpha_{N-j}=\cos 2 \alpha_{N-j+1}, \cos 2 \alpha_{N-1}=\left(\cos 2 \alpha_{0}\right)^{N}$. Within the first stage, the state changes as

$$
\left\{\prod_{j=2} \prod_{N} \mathrm{D}_{j}\left(\alpha_{0}, \alpha_{N-j}\right)\right\}\left|\varphi_{ \pm}\left(\alpha_{0}\right)\right\rangle^{\otimes N}=\left|\varphi_{ \pm}\left(\alpha_{N-1}\right)\right\rangle_{1} \otimes|0\rangle^{\otimes(N-1)}
$$




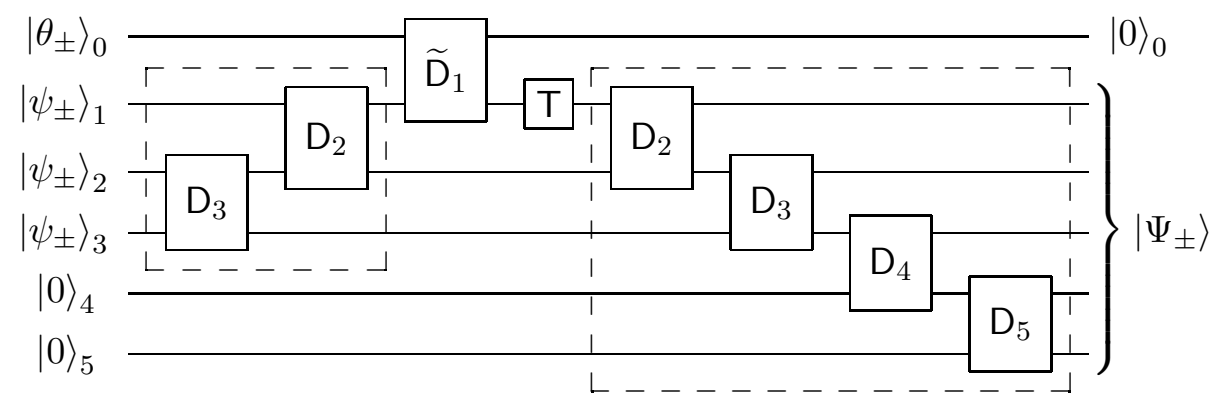

FIG. 1: The circuit for optimal $3 \rightarrow 5$ cloning with the a priori information. For brevity, the gate $\mathrm{D}_{j}\left(\alpha_{0}, \alpha_{N-j}\right)$ in the left box and the gate $\mathrm{D}_{k}\left(\alpha_{0}, \alpha_{L-k}\right)$ the right box are both denoted as $\mathrm{D}_{k}$.

where the gates $\mathrm{D}_{j}$ are put from right to left with decreasing $j$. This part transfers a total distinguishability of the $N$ originals $\left|\varphi_{ \pm}\left(\alpha_{0}\right)\right\rangle$ into the one-qubit state $\left|\varphi_{ \pm}\left(\alpha_{N-1}\right)\right\rangle$. An example for $3 \rightarrow 5$ cloning is shown on Fig. 1 .

For using an a priori information, we now include the turned gate $\widetilde{\mathrm{D}}_{1}$. This gate transfers distinguishability of ancilla's states $\left|\theta_{ \pm}\right\rangle$to those of qubit 1 , namely

$$
\widetilde{\mathrm{D}}_{1}\left(\theta, \alpha_{N-1}\right)\left|\varphi_{ \pm}(\theta)\right\rangle_{0} \otimes\left|\varphi_{ \pm}\left(\alpha_{N-1}\right)\right\rangle_{1}=|0\rangle_{0} \otimes\left|\varphi_{ \pm}\left(\theta_{1}\right)\right\rangle_{1} .
$$

After the action of gate $\widetilde{\mathrm{D}}_{1}$, the ancilla contains no information about distinguishability. All the distinguishability of inputs are now concentrated on two possible states $\left|\varphi_{ \pm}\left(\theta_{1}\right)\right\rangle$ of qubit 1 , where $\cos 2 \theta_{1}=\cos 2 \theta \cos 2 \alpha_{N-1}$. Now the scheme acts on the qubit 1 by the unitary operator $T$ specified as

$$
\mathrm{T}\left|\varphi_{ \pm}\left(\theta_{1}\right)\right\rangle=\mu_{ \pm}\left|\varphi_{+}\left(\alpha_{L-1}\right)\right\rangle+\nu_{ \pm}\left|\varphi_{-}\left(\alpha_{L-1}\right)\right\rangle .
$$

The values of angle $\alpha_{L-1}$ and complex numbers $\mu_{ \pm}$and $\nu_{ \pm}$will be found below. So the second stage results in the final state

$$
\left|\Phi_{ \pm}\right\rangle=\mu_{ \pm}\left|\varphi_{+}\left(\alpha_{L-1}\right)\right\rangle_{1} \otimes|0\rangle^{\otimes(L-1)}+\nu_{ \pm}\left|\varphi_{-}\left(\alpha_{L-1}\right)\right\rangle_{1} \otimes|0\rangle^{\otimes(L-1)}
$$

of the $L$ qubits. In Fig. 1, the gates $\widetilde{\mathrm{D}}_{1}$ and $\mathrm{T}$ between the dash boxes perform the second stage. Its structure is independent of numbers $N$ and $L$. Note that this stage and an a priori information are not considered in Ref. [17].

We put two linear combinations of the ideal outputs $\left|\psi_{+}\right\rangle^{\otimes L}$ and $\left|\psi_{-}\right\rangle^{\otimes L}$ as

$$
\left|\Psi_{ \pm}\right\rangle:=\mu_{ \pm}\left|\varphi_{+}\left(\alpha_{0}\right)\right\rangle^{\otimes L}+\nu_{ \pm}\left|\varphi_{-}\left(\alpha_{0}\right)\right\rangle^{\otimes L} .
$$

The final stage of cloning is posed as $\left|\Phi_{ \pm}\right\rangle \longmapsto\left|\Psi_{ \pm}\right\rangle$. Let us continue the sequence $\left\{\alpha_{0}, \alpha_{1}, \ldots, \alpha_{N-1}\right\}$ with respect to the above recurrence, that is

$$
\cos 2 \alpha_{L-k+1}=\cos 2 \alpha_{0} \cos 2 \alpha_{L-k},
$$

where $\alpha_{L-k} \in[0 ; \pi / 4]$. Hence we obtain $\cos 2 \alpha_{L-1}=\left(\cos 2 \alpha_{0}\right)^{L}$. Due to the property (7.2) of distinguishability transfer gate, we have

$$
\mathrm{D}_{k}\left(\alpha_{0}, \alpha_{L-k}\right)\left|\varphi_{ \pm}\left(\alpha_{L-k+1}\right)\right\rangle_{k-1} \otimes|0\rangle_{k}=\left|\varphi_{ \pm}\left(\alpha_{0}\right)\right\rangle_{k-1} \otimes\left|\varphi_{ \pm}\left(\alpha_{L-k}\right)\right\rangle_{k}
$$

In the third stage, the label $k$ in (6.9) runs from $k=2$ to $k=L$. So, the gate $\mathrm{D}_{2}\left(\alpha_{0}, \alpha_{L-2}\right)$ acts on the qubits 1 and 2 , the gate $\mathrm{D}_{3}\left(\alpha_{0}, \alpha_{L-3}\right)$ acts on the qubits 2 and 3 , and so on. The total action is described by

$$
\left\{{ }_{L} \prod_{k=2} \mathrm{D}_{k}\left(\alpha_{0}, \alpha_{N-k}\right)\right\}\left|\varphi_{+}\left(\alpha_{L-1}\right)\right\rangle_{1} \otimes|0\rangle^{\otimes(L-1)}=\left|\varphi_{ \pm}\left(\alpha_{0}\right)\right\rangle^{\otimes L}
$$

where the gates $\mathrm{D}_{k}$ are put from right to left with increasing $k$. In (6.10), the accumulated distinguishability is distributed among the $L$ qubits of interest. On Fig. 1, the four gates $D_{2}, D_{3}, D_{4}$ and $D_{5}$ of the third stage are grouped in the right dash box. Using the linearity, we see that $\left|\Phi_{ \pm}\right\rangle \longmapsto\left|\Psi_{ \pm}\right\rangle$too. Due to $\left\langle\Phi_{+} \mid \Phi_{-}\right\rangle=\left\langle\Psi_{+} \mid \Psi_{-}\right\rangle$,

$$
\left(\mu_{+}^{*} \nu_{-}+\nu_{+}^{*} \mu_{-}\right) \cos 2 \alpha_{L-1}=\left(\mu_{+}^{*} \nu_{-}+\nu_{+}^{*} \mu_{-}\right)\left(\cos 2 \alpha_{0}\right)^{L},
$$

that is actually correct. Specifying concrete values of $\mu_{ \pm}$and $\nu_{ \pm}$and herewith the single-qubit gate T in Eq. (6.5), we can optimize either the relative error or the global fidelity. In each case, we superpose the $\operatorname{span}\left\{\left|\varphi_{+}\left(\theta_{1}\right)\right\rangle,\left|\varphi_{-}\left(\theta_{1}\right)\right\rangle\right\}$ 
onto the $\operatorname{span}\left\{\left|\varphi_{+}\left(\alpha_{L-1}\right)\right\rangle,\left|\varphi_{-}\left(\alpha_{L-1}\right)\right\rangle\right\}$. Then after the second stage the $L$ qubits of interest lie in the states $\left|\Phi_{ \pm}\right\rangle$. For the optimality with respect to the relative error, we demand that $\delta_{+}^{\prime}=0$, whence we get $\left|\Psi_{+}\right\rangle=\left|\varphi_{+}\left(\alpha_{0}\right)\right\rangle^{\otimes L}$ and $\mu_{+}=1, \nu_{+}=0$ from (6.7). The angle between $\left|\varphi_{+}\left(\theta_{1}\right)\right\rangle$ and $\left|\varphi_{-}\left(\theta_{1}\right)\right\rangle$ is equal to $2 \theta_{1}$, the angle between $\left|\varphi_{+}\left(\alpha_{L-1}\right)\right\rangle$ and $\left|\varphi_{-}\left(\alpha_{L-1}\right)\right\rangle$ is equal to $2 \alpha_{L-1}>2 \theta_{1}$. Because unitary transformations preserve angles, the angle between T $\left|\varphi_{-}\left(\theta_{1}\right)\right\rangle$ and $\left|\varphi_{-}\left(\alpha_{L-1}\right)\right\rangle$ is equal to $\delta_{-}^{\prime}=2 \alpha_{L-1}-2 \theta_{1}$ Within the third stage, the state $\left|\Phi_{-}\right\rangle=\mathrm{T}\left|\varphi_{-}\left(\theta_{1}\right)\right\rangle_{1} \otimes|0\rangle^{\otimes(L-1)}$ maps to $\left|\Psi_{-}\right\rangle$. By definition, the value $\delta_{-}^{\prime}$ is angle between $\left|\Psi_{-}\right\rangle$and $\left|\varphi_{-}\left(\alpha_{0}\right)\right\rangle^{\otimes L}=\left|\psi_{-}\right\rangle^{\otimes L}$. Since $\cos \Delta_{ \pm}^{(L)}=\left(\cos 2 \alpha_{0}\right)^{L}=$ $\cos 2 \alpha_{L-1}$ and $\cos \varkappa_{ \pm}=\left(\cos 2 \alpha_{0}\right)^{N} \cos 2 \theta=\cos 2 \theta_{1}$, we find the needed value $\delta_{-}^{\prime}=\Delta_{ \pm}^{(L)}-\varkappa_{ \pm}$. Thus, the inequality (3.3) is saturated too, and the built scheme is really optimal with respect to the relative error.

Note that $\mu_{-}$and $\nu_{-}$are found as $\mu_{-}=\sin \left(\Delta_{ \pm}^{(L)}-\varkappa_{ \pm}\right) / \sin \Delta_{ \pm}^{(L)}$ and $\nu_{-}=\sin \varkappa_{ \pm} / \sin \Delta_{ \pm}^{(L)}$. But the described geometrical picture is quite sufficient for all the purposes. In the same manner, the optimization of cloning with respect to the global fidelity would be considered. As result, the generalization of the deterministic cloner of Ref. [17] to prior ancillary information can be obtained.

\section{CONCLUSION}

We have analyzed a new optimality criterion for the state-dependent cloning of several states with arbitrary prior probabilities and an ancillary information. The notion of the relative error has been extended to the general cloning scenario. The lower bounds on the relative error have been obtained for both the two-state and multi-state cases. The attainability of the derived bounds has been discussed. The quantum circuit for optimal cloning of two pure states with respect to the relative error has been built. Our approach is based on the simple geometrical description, which generally clarifies origins of a bound for one or another figure of merit. In principle, the described scheme allows to develop cloning circuit that is optimal with respect to any non-local figure of merit. The scenario with an $a$ priori information in the ancilla was inspired by the stronger no-cloning theorem. The obtained conclusions on a possible merit of the cloning contribute to this subject. Unequal prior probabilities of inputs are usual in communication systems. The examination of mixed-state cloning is needed because all the real devices are inevitably exposed to noise. Analysis with respect to the relative error may have potential applications to the problem of eavesdropping in quantum cryptography.

\section{Appendix A. Lemma}

Let us consider the function $f(x, y)=p \sin x+q \sin y$, where positive $p$ and $q$ obey $p+q=1$. Let $a \in[0 ; \pi / 2]$ be a fixed parameter. The range of variables is stated by conditions $x+y \geq a, 0 \leq x \leq \pi / 2$ and $0 \leq y \leq \pi / 2$. This domain $D$ is a square whose left-lower corner is cut off by line $x+y=a$.

Lemma 4 The global minimum of the function $f(x, y)$ in the domain $D$ is equal to $f_{\min }=\min \{p, q\} \sin a$.

Proof Inside of the domain $D$, we have $\partial f / \partial x \neq 0$ and $\partial f / \partial y \neq 0$. So the extreme values are reached on the boundary $\partial D$. Consider those segments that are parallel to either axis $x$ or axis $y$. The minimum value on these segments is equal to either $f(a, 0)=p \sin a$ or $f(0, a)=q \sin a$, i.e. $\min \{p, q\} \sin a$.

On the segment $x+y=a$, we put $x=a / 2+t$ and $y=a / 2-t$ with $t \in[-a / 2 ; a / 2]$, whence $f(x, y)=$ $\sin (a / 2) \cos t+(p-q) \cos (a / 2) \sin t$. By calculus, we obtain the extreme value $\left(p^{2}-2 p q \cos a+q^{2}\right)^{1 / 2}$ for $t \in[-a / 2 ; a / 2]$. This value is not less than both the $p \sin a$ and $q \sin a$.

\section{Appendix B. Distinguishability transfer gate}

By this operation, a distiguishability of the possible states of second qubit is translated to those of the first. It is convenient to introduce a family of states $\left|\varphi_{ \pm}(\alpha)\right\rangle:=\cos \alpha|0\rangle \pm \sin \alpha|1\rangle$ with the inner product $\left\langle\varphi_{+}(\alpha) \mid \varphi_{-}(\alpha)\right\rangle=\cos 2 \alpha$, where $\alpha \in[0 ; \pi / 4]$. As is well-known, one- and two-qubit gates are sufficient to implement universal computation. In the context of cloning, the writers of Ref. [17] note that only one type of pair-wise interaction is needed. The distiguishability transfer gate is described by [17]

$$
\begin{aligned}
& \mathrm{D}(\alpha, \beta)\left|\varphi_{ \pm}(\alpha)\right\rangle_{1} \otimes\left|\varphi_{ \pm}(\beta)\right\rangle_{2}=\left|\varphi_{ \pm}(\gamma)\right\rangle_{1} \otimes|0\rangle_{2} \\
& \mathrm{D}(\alpha, \beta)\left|\varphi_{ \pm}(\gamma)\right\rangle_{1} \otimes|0\rangle_{2}=\left|\varphi_{ \pm}(\alpha)\right\rangle_{1} \otimes\left|\varphi_{ \pm}(\beta)\right\rangle_{2}
\end{aligned}
$$

where by the unitarity $\cos 2 \alpha \cos 2 \beta=\cos 2 \gamma$. It follows from Eqs. (7.1) and (7.2) that the operation D is Hermitian [17]. The action of distiguishability transfer gate on two-qubit register is shown on Figure 2. The corresponding circuit of CNOT elements and one-qubit operations is given in Ref. [17]. 


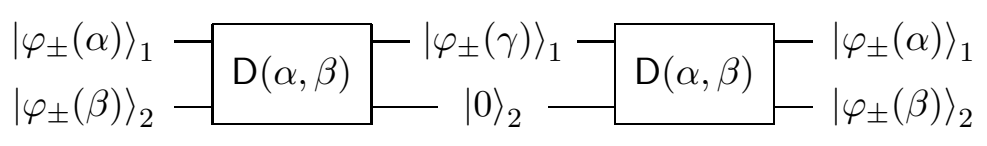

FIG. 2: The action of distiguishability transfer gate.

[1] M. Hayashi, Quantum Information: An Introduction (Springer, Berlin, 2006).

[2] N. Gisin et al., Rev. Mod. Phys. 74 (2002) 145-195.

[3] A. M. Childs and W. van Dam, Rev. Mod. Phys. 82 (2009) 1-52.

[4] A. K. Ekert et al., Phys. Rev. A 50 (1994) 1047-1056.

[5] S. Pirandola, Int. J. Quantum Inf. 6 (2008) 765-771.

[6] W. K. Wootters and W. Zurek, Nature 299 (1982) 802-803.

[7] D. Dieks, Phys. Lett. A 92 (1982) 271-272.

[8] H. Barnum et al., Phys. Rev. Lett. 76 (1996) 2818-2821.

[9] V. Bužek and M. Hillery, Phys. Rev. A 54 (1996) 1844-1852.

[10] N. J. Cerf and J. Fiurášek, Optical quantum cloning - a review, quant-ph/0512172.

[11] H. Fan, Quantum cloning machines, in Quantum Computation and Information. From Theory to Experiment, eds. H. Imai and M. Hayashi (Springer, Berlin, 2006), 63-110.

[12] M. Hillery and V. Bužek, Phys. Rev. A 56 (1997) 1212-1216.

[13] D. Bruß et al., Phys. Rev. A 57 (1998) 2368-2378.

[14] S. Adhikari et al., Quantum Inf. Process. 6 (2007) 197-219.

[15] M. Siomau and S. Fritzsche Eur. Phys. J. D 57 (2010) 293-300

[16] A. E. Rastegin, Phys. Rev. A 66 (2002) 042304.

[17] A. Chefles and S. M. Barnett, Phys. Rev. A 60 (1999) 136-144.

[18] Y. J. Han et al., Phys. Rev. A 66 (2002) 052301.

[19] A. Ya. Kazakov, Int. J. Quantum Inf. 8 (2010) 435-442.

[20] C. H. Bennett, Phys. Rev. Lett. 68 (1992) 3121-3124.

[21] A. E. Rastegin, Phys. Rev. A 67 (2003) 012305.

[22] J. A. Miszczak et al., Quantum Information \& Computation 9 (2009) 0103-0130.

[23] A. E. Rastegin, J. Opt. B: Quantum Semiclassical Opt. 5 (2003) S647-S650.

[24] R. Jozsa, A stronger no-cloning theorem, quant-ph/0204153.

[25] A. E. Rastegin, Phys. Rev. A 68 (2003) 032303.

[26] Z. H. Ma et al., Phys. Lett. A 373 (2009) 3407-3409.

[27] A. E. Rastegin, Sine distance for quantum states, quant-ph/0602112.

[28] A. Uhlmann, Rep. Math. Phys. 9 (1976) 273-279.

[29] R. Jozsa, J. Mod. Optics 41 (1994) 2315-2323.

[30] A. E. Rastegin, J. Phys. A: Math. Theor. 40 (2007) 9533-9549.

[31] A. E. Rastegin, Quantum Inf. Process. 9 (2010) 61-73.

[32] I. D. Ivanovic, Phys. Lett. A 123 (1987) 257-259.

[33] A. Peres, Phys. Lett. A 128 (1988) 19.

[34] C. W. Helstrom, Quantum Detection and Estimation Theory (Academic Press, New York, 1976).

[35] L.-M. Duan and G.-C. Guo, Phys. Lett. A 243 (1998) 261-264.

[36] A. Chefles and S. M. Barnett, J. Phys. A: Math. Gen. 31 (1998) 10097-10103. 